\title{
A Network Theory of Nonlinear Viscoelasticity. II. Steady Simple Elongation and Periodic Shear Deformation Superposed on Steady Shear Flow
}

\author{
Shizuo HAYASHI \\ Department of Physics, Faculty of General Studies, \\ Gunma University, Aramaki, Maebashi 371, Japan.
}

(Received December 5, 1980)

\begin{abstract}
The theory of nonlinear viscoelasticity presented in a previous report [Polymer Jounrnal, 10, 59-67 (1978)] is applied to certain deformations not yet treated previously. Tensile viscosity increases with increasing elongation speed and tensile stress growth at the onset of elongational flow is monotonous. The storage and loss moduli for a periodic deformation superposed on a steady shear flow are predicted. For parallel superposition, the storage modulus vanishes at a low frequency when the shear rate of the steady flow is high, but the loss modulus does not vanish over the entire frequency region, though it shows a nonlinear character. The viscosity obtained from the dynamic viscosity for the periodic deformation is smaller than the viscosity of steady flow. For an orthogonal superposition, the storage and loss moduli show the same frequency dependence as that in the linear case and the shear rate dependence of moduli at a fixed frequency is the same as that of non-Newtonian viscosity.

KEY WORDS Nonlinear Viscoelasticity / Network Theory / Elongational Flow / Superposition / Steady Shear Flow / Periodic Deformation / Dynamic Modulous /
\end{abstract}

In previous reports, ${ }^{1,2}$ we proposed a constitutive equation of nonlinear viscoelasticity using a temporarily crosslinked network model and applied it to certain typical deformations in relation to the non-Newtonian viscosity of steady shear flow, the stress growth at the onset of a shear flow with constant shear rate, the nonlinear stress relaxation at a constant large deformation and the stress relaxation at a sudden termination of steady shear flow. In this paper, the theory is applied to other typical deformations.

We briefly summarize the equations given in the previous report. ${ }^{1,2}$ Let the deformation tensor at a given time $t$ be $\gamma(t) \equiv \gamma$, the relative deformation tensor at a time $t$ with respect to a previous time $t^{\prime}$ be $\gamma\left(t, t^{\prime}\right)=\gamma(t) \cdot \gamma^{-1}\left(t^{\prime}\right)$ and the average internal strain tensor or the strain tensor of each chain in the network be $\alpha(t) \equiv \alpha$. The fundamental slip equation giving the relation between the internal strain and the deformation is

$$
\dot{\gamma} \cdot \gamma^{-1} \cdot \boldsymbol{\alpha}+\alpha \cdot \gamma^{+-1} \cdot \dot{\gamma}^{+}-\dot{\alpha}=\frac{1}{\tau_{0}}(\boldsymbol{\alpha}-\mathbf{1})
$$

$$
\alpha(t)=\int_{-\infty}^{t} \frac{\mathrm{e}^{-\left(t-t^{\prime}\right) / \tau_{0}}}{\tau_{0}} \gamma\left(t, t^{\prime}\right) \cdot \gamma^{+}\left(t, t^{\prime}\right) \mathrm{d} t^{\prime}
$$

where the dot refers to differentiation with respect to time, the dagger is the transpose of tensors, and $\tau_{0}$ is the relaxation time of the slip process. The relaxation process due to the slip of chains is assumed to be characterized by a single relaxation time, $\tau_{0}$.

Let the number of chains in unit volume of the network be $v$. The change in the number of chains with time is given by

$$
\begin{gathered}
\frac{\mathrm{d} v}{\mathrm{~d} t}=-k(t) v+g(t) \\
v(t)=\exp \left\{-\int_{0}^{t} k\left(t^{\prime}\right) \mathrm{d} t^{\prime}\right\} \\
\times\left[v_{0}+\int_{0}^{t}\left(g(t) \exp \int_{0}^{t^{\prime}} k\left(t^{\prime \prime}\right) \mathrm{d} t^{\prime \prime}\right) \mathrm{d} t^{\prime}\right]
\end{gathered}
$$

where $k(t)$ is the chain breakage coefficient at time $t$ and $g(t)$ is the rate of chain creation per unit time, 
the chain generating function. If we attach the subscript $e$ to quantities in the stress-free state, $k_{\mathrm{e}} v_{\mathrm{e}}=g_{\mathrm{e}}$ since the rates of chain breakage and chain creation are equal at the stress-free state. For $g(t)$ and $k(t)$ in a deformed state, we assume the relation, ${ }^{1}$

$$
\left.\begin{array}{l}
g(t)=g_{\mathrm{e}}=\text { constant } \\
k(t)=k_{\mathrm{e}}(\operatorname{Tr} \alpha / 3)^{1 / 2}
\end{array}\right\}
$$

and obtain the stress as

$$
\begin{aligned}
\boldsymbol{\sigma}(t)=v(t) k_{\mathrm{B}} T \boldsymbol{\alpha}-p \mathbf{1} \\
\boldsymbol{\sigma}(t)=k_{\mathrm{B}} T\left[\exp \left\{-\int_{0}^{t} k\left(t^{\prime}\right) \mathrm{d} t^{\prime}\right\}\right. \\
\left.\times\left\{v_{0}+g_{\mathrm{e}} \int_{0}^{t} \exp \int_{0}^{t^{\prime}} k\left(t^{\prime \prime}\right) \mathrm{d} t^{\prime \prime}\right\}\right] \\
\times \int_{-\infty}^{t} \frac{\mathrm{e}^{-\left(t-t^{\prime}\right) / \tau_{0}}}{\tau_{0}} \gamma\left(t, t^{\prime}\right) \cdot \gamma^{+}\left(t, t^{\prime}\right) \mathrm{d} t^{\prime}-p \mathbf{1}
\end{aligned}
$$

where $k_{\mathrm{B}}$ is the Boltzmann constant.

\section{STRESS GROWTH OF VERY HIGH MOLECULAR-WEIGHT-SYSTEM AT THE ONSET OF STEADY SHEAR FLOW}

We suggested in a previous report ${ }^{1,2}$ that the strength of overshoot in the stress growth decreases as the molecular weight increases and depends on the chain-breakage rate. The chain-breakage rate at a constant shear rate decreases as the number of chain terminals decreases; that is, as the molecular chain length increases, since chain breakage occurs at the terminals of the molecular chains. If the molecular weight is very high, $\mathrm{d} v / \mathrm{d} t$ is very small and $v$ is approximately expressed by $v \simeq v_{\mathrm{e}} k_{\mathrm{e}} / k(t)$, and the stress growth at the onset of a steady flow is given by eq $23-26$ in the previous report. ${ }^{1}$ That is,

$$
\begin{gathered}
\sigma_{12}(t, \dot{\gamma})=v_{\mathrm{e}} k_{\mathrm{B}} T \tau_{0} \dot{\gamma} F_{0}(t)\left(1-\mathrm{e}^{-t / \tau_{0}}\right) \\
F_{0}(t)=\left[1+\frac{2}{3} \tau_{0}^{2} \dot{\gamma}^{2}\left\{1-\mathrm{e}^{-t / \tau_{0}}\left(1+\frac{t}{\tau_{0}}\right)\right\}\right]^{1 / 2}
\end{gathered}
$$

The $\tilde{\eta}(t, \gamma)=\sigma_{12}(t, \gamma) / \gamma$ versus $t / \tau_{0}$ relation obtained from eq 5 and 6 is shown on the logarithmic scale in Figure 1, where the number on each curve is the value of the parameter $\sqrt{2 / 3} \tau_{0} \dot{\gamma}$. The predicted curves have striking resemblance to the experiment-

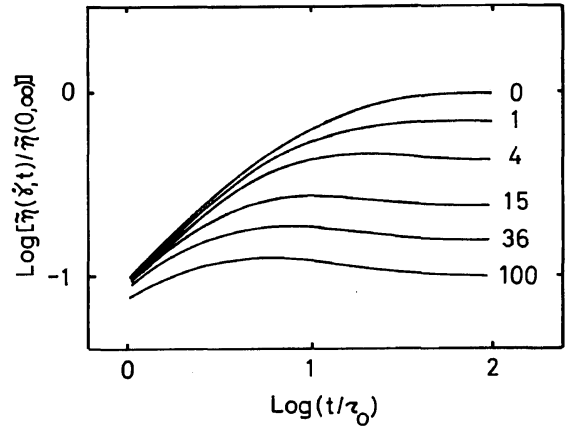

Figure 1. The stress growth at the onset of shear flow for a system with very long chain molecules. The number on each curve indicates the value of the parameter $\sqrt{2 / 3}$ $\dot{\gamma} \tau_{0}$. The stress overshoot is not so clear even though the system shows the evident non-Newtonian viscosity.

al data obtained by Osaki et al. ${ }^{3}$ Though the curves show no distinct stress overshoot, they do show a non-Newtonian viscosity since $\tilde{\eta}(t, \dot{\gamma})$, for a long period of time, is a decreasing function of $\gamma$. Therefore, so far as the present theory is concerned, we may predict that the network structure showing large non-Newtonian visocisty does not always show an evident stress overshoot at the stress growth. The quantitative relationship between the strength of overshoot and the molecular weight is not clear.

\section{TENSILE VISCOSITY}

When a stationary simple elongation is given on a system, the stationary condition is expressed by,

$$
\frac{\mathrm{d}}{\mathrm{d} t}\left(\dot{\gamma} \cdot \gamma^{-1}\right)=0
$$

and only the diagonal elements of $\gamma$ are non-zero:

$$
\gamma=\left(\begin{array}{ccc}
\gamma & 0 & 0 \\
0 & \gamma^{\prime} & 0 \\
0 & 0 & \gamma^{\prime}
\end{array}\right)
$$

Let $s$ be Poisson's ratio defined by $s=-\ln \gamma^{\prime} / \ln \gamma$ and $a$ be the elongation speed defined by $a=\dot{\gamma} / \gamma$. We obtain

$$
\gamma(t)=\left(\begin{array}{lll}
\mathrm{e}^{a t} & 0 & 0 \\
0 & \mathrm{e}^{-s a t} & 0 \\
0 & 0 & \mathrm{e}^{-s a t}
\end{array}\right)
$$




$$
\boldsymbol{\sigma}(t)=\left(\begin{array}{ccc}
\left(1-2 a \tau_{0}\right)^{-1} & 0 & 0 \\
0 & \left(1+2 s a \tau_{0}\right)^{-1} & 0 \\
0 & 0 & \left(1+2 s a \tau_{0}\right)^{-1}
\end{array}\right)
$$

From the relation $k=k_{\mathrm{e}}[\operatorname{Tr}(\alpha / 3)]^{1 / 2}$ given by eq $3, v$ becomes

$$
\begin{aligned}
v & =v_{\mathrm{e}}[\operatorname{Tr}(\boldsymbol{\alpha} / 3)]^{1 / 2} \\
& =v_{\mathrm{e}}\left[\left\{\left(1-2 a \tau_{0}\right)^{-1}+2\left(1+2 s a \tau_{0}\right)^{-1}\right\} / 3\right]^{-1 / 2}
\end{aligned}
$$

If the stretching force acts only in the elongational direction, $\sigma$ has the form

$$
\boldsymbol{\sigma}=\left(\begin{array}{lll}
\sigma & 0 & 0 \\
0 & 0 & 0 \\
0 & 0 & 0
\end{array}\right)
$$

and, from eq 5 , $\sigma$ becomes,

$$
\sigma=v k_{\mathrm{B}} T\left[\left(1-2 a \tau_{0}\right)^{-1}-\left(1+2 s a \tau_{0}\right)^{-1}\right]
$$

The tensile viscosity $\eta_{\mathrm{E}}$ is defined by,

$$
\eta_{\mathrm{E}}=\sigma / a
$$

and the ratio $\eta_{\mathrm{E}} / \eta_{\mathrm{so}}$ of $\eta_{\mathrm{E}}$ to the zero-shear rate viscosity $\eta_{\mathrm{so}}$ is

$$
\frac{\eta_{\mathrm{E}}}{\eta_{\mathrm{so}}}=\frac{\sqrt{3}\left[\left(1-2 a \tau_{0}\right)^{-1}-\left(1+2 s a \tau_{0}\right)^{-1}\right]}{a \tau_{0}\left[\left(1-2 a \tau_{0}\right)^{-1}+\left(1+2 s a \tau_{0}\right)^{-1}\right]^{1 / 2}}
$$

The $\eta_{\mathrm{E}} / \eta_{\mathrm{so}}$ versus a $\tau_{0}$ relation for $s=1 / 2$ calculated from eq 15 is shown in Figure 2; Trouton's relation is satisfied for small $a$. The value of $\eta_{\mathrm{E}} / \eta_{\mathrm{so}}$ increases as the elongation speed increases: this is not always in agreement with experimental results. ${ }^{1-14}$

When $a \tau_{0}$ approaches $1 / 2, \eta_{\mathrm{E}} / \eta_{\mathrm{so}}$ becomes very large. This is due to the large stress or large elongational deformation of chains. However, since the present theory has been formulated by assuming Gaussian chains, it should not be applied to a case in which elongation speed is so large that chains are greatly deformed.

\section{STRESS GROWTH AT THE ONSET OF STEADY SIMPLE ELONGATIONAL FLOW}

When a steady simple elongational flow (the elongational rate designated as a) starts at $t=0$, $\gamma$ is expressed as

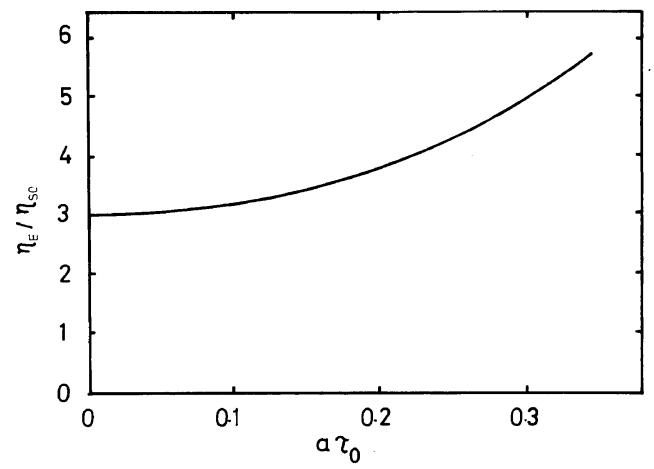

Figure 2. Tensile viscosity versus elongation speed for elongational flow, where $\eta_{\text {so }}$ is zero shear viscosity. The tensile viscosity increases with the elongation speed, but the rate of increase is smaller than that obtained in the linear range of this theory.

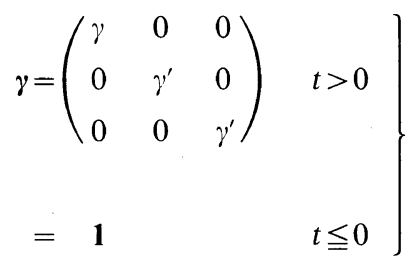

and $\gamma\left(t, t^{\prime}\right)$ becomes,

$$
\begin{aligned}
\gamma\left(t, t^{\prime}\right) & =\left(\begin{array}{lll}
\mathrm{e}^{a t} & 0 & 0 \\
0 & \mathrm{e}^{-s a t} & 0 \\
0 & 0 & \mathrm{e}^{-s a t}
\end{array}\right) \begin{array}{l}
t>0 \\
t^{\prime} \leqq 0
\end{array} \\
& =\left(\begin{array}{lll}
\mathrm{e}^{a\left(t-t^{\prime}\right)} & 0 & 0 \\
0 & \mathrm{e}^{-s a\left(t-t^{\prime}\right)} & 0 \\
0 & 0 & \mathrm{e}^{-s a\left(t-t^{\prime}\right)}
\end{array}\right) \begin{array}{l}
t>0 \\
t^{\prime} \geqq 0
\end{array} \\
& =1
\end{aligned}
$$


From eq 3 and 17 we obtain,

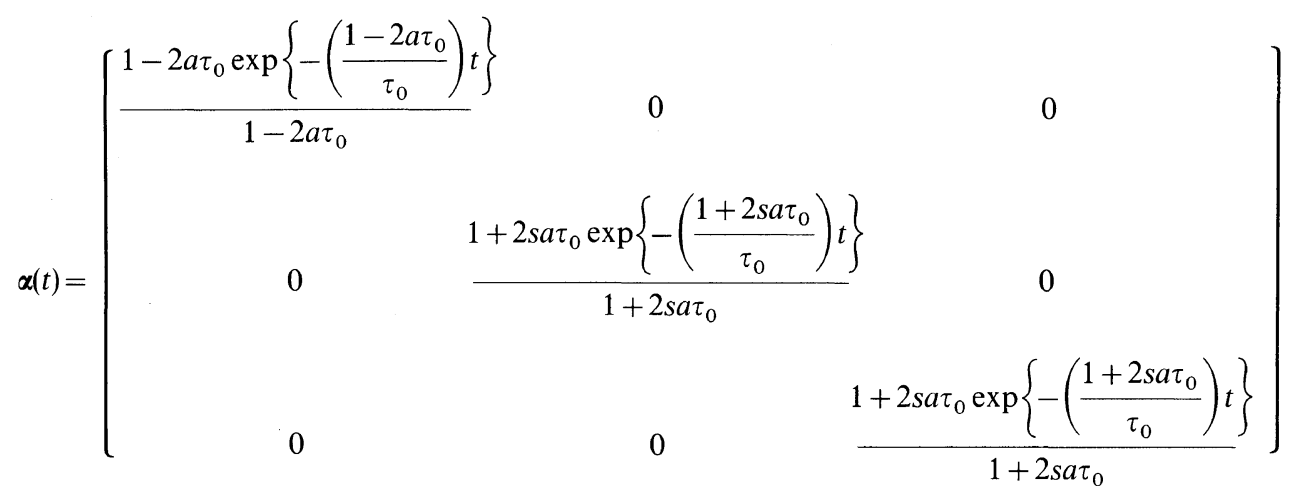

and,

$$
k(t)=\frac{1}{\sqrt{3}}\left[\frac{1-2 a \tau_{0} \exp \left\{-\left(\frac{1-2 a \tau_{0}}{\tau_{0}}\right) t\right\}}{1-2 a \tau_{0}}+2 \frac{1+2 s a \tau_{0} \exp \left\{-\left(\frac{1+2 s a \tau_{0}}{\tau_{0}}\right) t\right\}}{1+2 s a \tau_{0}}\right]^{1 / 2}
$$

The stress tensor $\sigma$ has the same formula as eq 12 and is given by

$$
\sigma(t)=v(t) k_{\mathrm{B}} T\left[\frac{1-2 a \tau_{0} \exp \left\{-\left(\frac{1-2 a \tau_{0}}{\tau_{0}}\right) t\right\}}{1-2 a \tau_{0}}-\frac{1+2 s a \tau_{0} \exp \left\{-\left(\frac{1+2 s a \tau_{0}}{\tau_{0}}\right) t\right\}}{1+2 s a \tau_{0}}\right]
$$

where $v(t)$ is the number of chains calculated from eq 2 by the use of $k(t)$ in eq 19.

The stress growth obtained from eq 20 for three values of elongation speed is shown in Figure 3, where $k_{\mathrm{e}} \tau_{0}=0.2$ as in previous reports, ${ }^{1,2}$ the number on each curve is the value of $a \tau_{0}$. The stress increases monotonously with time and shows no stress overshoot.

Experimental tensile stress growth is complex and diverse. Some of the experimental results give stress growth curves that increase monotonously with time; some of the stress growth curves have concave shoulders ${ }^{7}$ and others have no shoulder but have growth rates which depend greatly on elongation speed. ${ }^{8-10}$ The other experimental results give stress growth curves, each having a maximum. ${ }^{12,14}$

The present theory explains only the monotonously increasing stress growth and fails to account for other complex experimental stress growths described above. Therefore, the present theory is imcomplete even qualitatively so far as the tensile stress growth is concerned. However, the complex and divergent features of the experi-

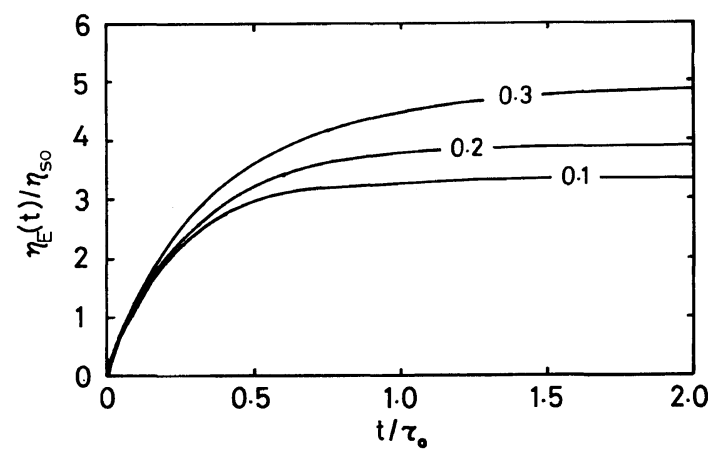

Figure 3. The tensile viscosity growth at the onset of elongational flow. The number on each curve indicates the value of the parameter $a \tau_{0}$. The growth is monotonous.

mental results ${ }^{6-14}$ show that further experiments are necessitated to confirm whether identical results are obtainable. 
A Network Theory of Nonlinear Viscoelasticity. II.

\section{PARALLEL SUPERPOSITION OF PERIODIC SHEAR DEFORMATION ON STEADY SHEAR FLOW}

When a parallel small periodic shear deformation is superposed on a steady shear flow with a constant shear rate $\dot{\gamma}$, the deformation tensor is given by

$$
\gamma(t)=\left(\begin{array}{ccc}
1 & \dot{\gamma} t+a_{0} \mathrm{e}^{i \omega t} & 0 \\
0 & 1 & 0 \\
0 & 0 & 1
\end{array}\right)
$$

and $\gamma\left(t, t^{\prime}\right)$ by

$$
\gamma\left(t, t^{\prime}\right)=\left(\begin{array}{ccc}
1 & \dot{\gamma}\left(t-t^{\prime}\right)+a_{0}\left(\mathrm{e}^{i \omega t}-\mathrm{e}^{i \omega t^{\prime}}\right) & 0 \\
0 & 1 & 0 \\
0 & 0 & 1
\end{array}\right)
$$

Neglecting terms higher than the first power of $a_{0}$, $a_{0}$ being very small compared with $\dot{\gamma} \tau_{0}$, we have from eq 1 ,

$$
\begin{aligned}
& \alpha_{12}(t)=\dot{\gamma} \tau_{0}+\frac{a_{0} i \omega \tau_{0} \mathrm{e}^{i \omega t}}{1+i \omega \tau_{0}} \\
& \alpha_{11}(t)=1+2 \dot{\gamma}^{2} \tau_{0}{ }^{2}+2 a_{0} \dot{\gamma} \tau_{0} \mathrm{e}^{i \omega t} \frac{i \omega \tau_{0}\left(2+i \omega \tau_{0}\right)}{\left(1+i \omega \tau_{0}\right)^{2}} \\
& \alpha_{22}=\alpha_{33}=1, \quad \alpha_{13}=\alpha_{31}=\alpha_{23}=\alpha_{32}=0
\end{aligned}
$$

and from eq 3 ,

$$
k(t)=k_{\mathrm{e}}\left[1+\frac{2}{3} \dot{\gamma}^{2} \tau_{0}{ }^{2}+\frac{2}{3} a_{0} \dot{\gamma} \tau_{0} \mathrm{e}^{i \omega t} \frac{i \omega \tau_{0}\left(2+i \omega \tau_{0}\right)}{\left(1+i \omega \tau_{0}\right)^{2}}\right]^{1 / 2}
$$

The number of chains $v^{*}(t)$ expressed in terms of the complex number is obtained from eq 2 as

$$
\begin{aligned}
v^{*}(t) & =\frac{v_{\mathrm{e}}}{h}\left[1-\frac{1}{3 h} \frac{\alpha \dot{\gamma} \tau_{0} i \omega \tau_{0}\left(2+i \omega \tau_{0}\right)}{\left(1+i \omega \tau_{0}\right)^{2}\left(\alpha h+i \omega \tau_{0}\right)} a_{0} \mathrm{e}^{i \omega t}\right] \\
h & \equiv h(\dot{\gamma})=\left(1+\frac{2}{3} \dot{\gamma}^{2} \tau_{0}^{2}\right)^{1 / 2} \\
\alpha & =k_{\mathrm{e}} \tau_{0}
\end{aligned}
$$

The complex shear stress $\sigma_{12}^{*}(\omega, t, \gamma)$ is given by

$$
\begin{aligned}
\sigma_{12}^{*}(\omega, t, \dot{\gamma}) & =v^{*}(t) k_{\mathrm{B}} T \alpha_{12}(t) \\
& =\frac{v_{\mathrm{e}} k_{\mathrm{B}} T}{h}\left[\dot{\gamma} \tau_{0}+\left\{\frac{i \omega \tau_{0}}{1+i \omega \tau_{0}}\right.\right.
\end{aligned}
$$

$$
\left.\left.-\frac{\alpha \dot{\gamma}^{2} \tau_{0}^{2} i \omega \tau_{0}\left(2+i \omega \tau_{0}\right)}{3 h\left(1+i \omega \tau_{0}\right)^{2}\left(\alpha h+i \omega \tau_{0}\right)}\right\} a_{0} \mathrm{e}^{i \omega t}\right]
$$

If we define the complex modulus corresponding to the small superposed periodic deformation by

$$
\tilde{G}_{\|}^{*}(\omega, \dot{\gamma})=\frac{\sigma_{12}^{*}(\omega, t, \dot{\gamma})-v_{\mathrm{e}} k_{\mathrm{B}} T \dot{\gamma} \tau_{0} / h}{a_{0} \mathrm{e}^{i \omega t}}
$$

we have

$\tilde{G}_{\|}^{*}(\omega, \dot{\gamma})=\frac{v_{\mathrm{e}} k_{\mathrm{B}} T i \omega \tau_{0}}{h\left(1+i \omega \tau_{0}\right)}\left[1-\frac{\alpha \dot{\gamma}^{2} \tau_{0}^{2}\left(2+i \omega \tau_{0}\right)}{3 h\left(1+i \omega \tau_{0}\right)\left(\alpha h+i \omega \tau_{0}\right)}\right]$

The storage and loss moduli for the superposed periodic part are obtained from eq 30 as follows:

$$
\begin{aligned}
\tilde{G}_{\|}{ }^{\prime}(\omega, \dot{\gamma})= & \frac{v_{\mathrm{e}} k_{\mathrm{B}} T \omega^{2} \tau_{0}{ }^{2}}{h\left(1+\omega^{2} \tau_{0}{ }^{2}\right)} \\
& \times\left[1-\frac{\alpha \dot{\gamma}^{2} \tau_{0}{ }^{2}}{3 h} \frac{\left\{2+\alpha h\left(3+\omega^{2} \tau_{0}{ }^{2}\right)\right\}}{\left(1+\omega^{2} \tau_{0}{ }^{2}\right)\left(\alpha^{2} h^{2}+\omega^{2} \tau_{0}{ }^{2}\right)}\right] \\
\tilde{G}_{\|}{ }^{\prime \prime}(\omega, \dot{\gamma})= & \frac{v_{\mathrm{e}} k_{\mathrm{B}} T \omega^{2} \tau_{0}{ }^{2}}{h\left(1+\omega^{2} \tau_{0}{ }^{2}\right)} \\
& \times\left[1-\frac{\alpha \dot{\gamma}^{2} \tau_{0}{ }^{2}}{3 h} \frac{\left\{2 \alpha h-\omega^{2} \tau_{0}{ }^{2}\left(3+\omega^{2} \tau_{0}{ }^{2}\right)\right\}}{\left(1+\omega^{2} \tau_{0}{ }^{2}\right)\left(\alpha^{2} h^{2}+\omega^{2} \tau_{0}{ }^{2}\right)}\right]
\end{aligned}
$$

The values of

$$
\lim _{\omega \rightarrow 0} \widetilde{G}_{\|}{ }^{\prime}(\omega, \dot{\gamma}) / \omega^{2}
$$

is negative for large $\dot{\gamma}$, whereas

$$
\lim _{\omega \rightarrow 0} G_{\|}{ }^{\prime \prime}(\omega, \dot{\gamma}) / \omega
$$

is always positive for all values of $\dot{\gamma}$.

The curves of $\tilde{G}_{\|}{ }^{\prime}(\omega, \gamma) / \omega^{2}$ and $\tilde{G}_{\|}{ }^{\prime \prime}(\omega, \gamma) / \omega$ against $\omega \tau_{0}$ calculated from eq 31 and 32 are shown in Figures 4 and 5 on a logarithmic scale, ${ }^{15}$ the number on each curve being the value of $\gamma \tau_{0}$. The frequency $\omega_{\mathrm{c}}$ at which $\widetilde{G}_{\|}{ }^{\prime}(\omega, \gamma) / \omega^{2}$ for a given $\dot{\gamma}$ vanishes is plotted in Figure 6.

Experimental evidence shows that nonlinearity appears more distinctly in $\widetilde{G}_{\|}{ }^{\prime}(\omega, \gamma) / \omega^{2}$ than in $\widetilde{G}_{\|}{ }^{\prime \prime}$ $(\omega, \gamma) / \omega^{15-18}$ and that $\tilde{G}_{\|}{ }^{\prime}(\omega, \gamma) / \omega^{2}$ vanishes at a low frequency for high $\gamma^{15,18}$ The theoretical results qualitatively agree with the experimental results.

When the zero frequency dynamic viscosity of the periodic part is denoted by $\eta_{\|}(\gamma)$, we have 


\section{S. HAYASHI}

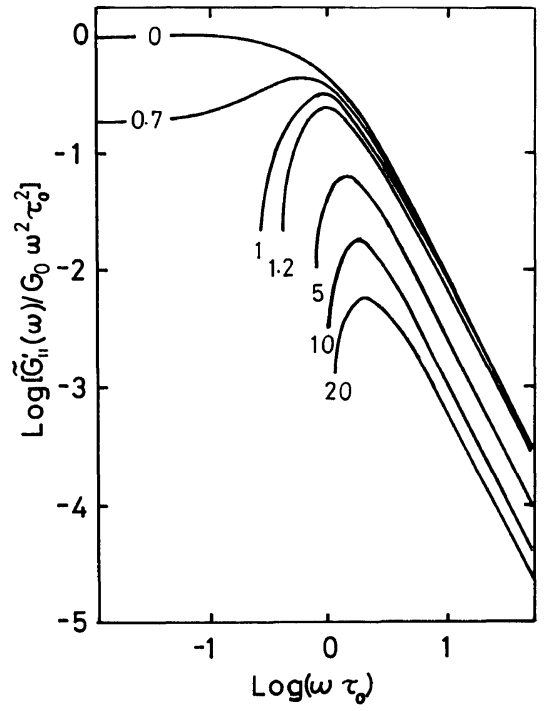

Figure 4. Storage modulus for the periodic part of a shear stress superposed in parallel on a steady flow. The number on each curve indicates the value of the parameter $\dot{\gamma} \tau_{0}$. The modulus vanishes at a critical frequency $\omega_{\mathrm{c}}$ for high shear rate.

$$
\begin{aligned}
& \eta_{\|}(\dot{\gamma})=\lim _{\omega \rightarrow 0} \frac{\tilde{G}_{\|}{ }^{\prime \prime}(\omega, \dot{\gamma})}{\omega}=\frac{\eta(\dot{\gamma})}{\left(1+2 / 3 \dot{\gamma}^{2} \tau_{0}{ }^{2}\right)} \\
& \eta(\dot{\gamma})=\frac{v_{\mathrm{e}} k_{\mathrm{B}} T \tau_{0}}{\left(1+2 / 3 \dot{\gamma}^{2} \tau_{0}{ }^{2}\right)^{1 / 2}}
\end{aligned}
$$

where, $\eta(\gamma)$ is the steady shear viscosity. From eq 33 and 34 we obtain

$$
\tilde{\eta}_{\|}(\dot{\gamma})=\eta(\dot{\gamma})\left[1+\frac{\mathrm{d} \ln \eta(\dot{\gamma})}{\mathrm{d} \ln \dot{\gamma}}\right]
$$

This equation has already been obtained through the phenomenological theory. ${ }^{20,21}$

The value of $\eta_{\|}(\gamma)$ is $\left(1+2 / 3 \gamma^{2} \tau_{0}^{2}\right)^{-1}$ times as large as $\eta(\gamma)$. This is because of the second term in the brackets of eq 32 and is associated with the fact that the stress induced by the interaction between the periodic change in the number of chains and the steady shear flow is of the first order of $a_{0}$.

\section{ORTHOGONAL SUPERPOSITION OF PERIODIC SHEAR DEFORMATION ON STEADY SHEAR FLOW}

When a small orthogonal periodic shear deformation is superposed on a steady shear flow with a

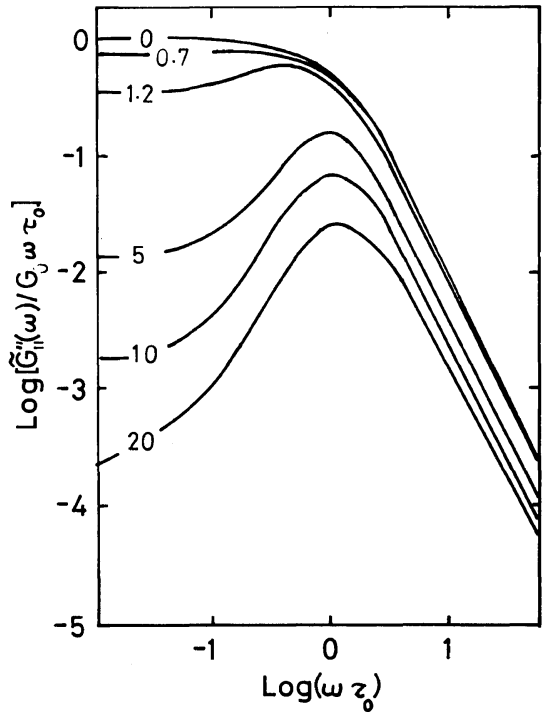

Figure 5. Loss modulus of the periodic part of shear stress superposed in parallel on a steady flow. Each number has the same meaning as in Figure 4. The modulus does not vanish for any frequency.

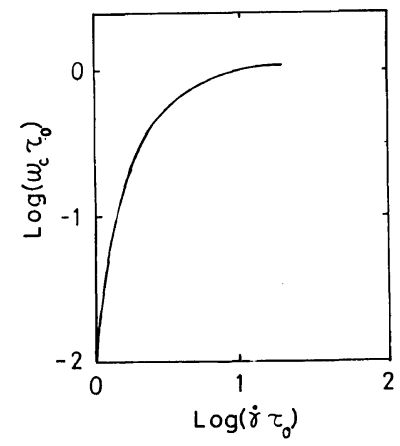

Figure 6. $\omega_{\mathrm{c}}$ versus $\dot{\gamma}$ relation obtained from Figure 4.

constant shear rate $\dot{\gamma}, \gamma(t)$ is given by,

$$
\gamma(t)=\left(\begin{array}{ccc}
1 & \dot{\gamma} t & 0 \\
0 & 1 & 0 \\
0 & a_{0} \mathrm{e}^{i \omega t} & 1
\end{array}\right)
$$

and $\gamma\left(t, t^{\prime}\right)$ by

$$
\gamma\left(t, t^{\prime}\right)=\left(\begin{array}{ccc}
1 & \dot{\gamma}\left(t-t^{\prime}\right) & 0 \\
0 & 0 & 0 \\
0 & a_{0}\left(\mathrm{e}^{i \omega t}-\mathrm{e}^{i \omega t^{\prime}}\right) & 1
\end{array}\right)
$$


Using the same approximation as that employed above, we obtain $\alpha(t)$ from eq 2 as

$$
\left.\begin{array}{l}
\alpha_{11}=1+2 \gamma^{2} \tau_{0}^{2} \\
\alpha_{12}=\alpha_{21}=\dot{\gamma} \tau_{0} \\
\alpha_{22}=\alpha_{33}=1 \\
\alpha_{23}=\alpha_{32}=\frac{i \omega \tau_{0}}{1+i \omega \tau_{0}} a_{0} \mathrm{e}^{i \omega t} \\
\alpha_{13}=\alpha_{31}=\dot{\gamma} \tau_{0} \frac{i \omega \tau_{0}\left(2+i \omega \tau_{0}\right)}{\left(1+i \omega \tau_{0}\right)^{2}} a_{0} \mathrm{e}^{i \omega t}
\end{array}\right\}
$$

and $k(t)$ from eq 3 as

$$
k=k_{\mathrm{e}} h=k_{\mathrm{e}}\left(1+2 / 3 \dot{\gamma}^{2} \tau_{0}{ }^{2}\right)^{1 / 2}
$$

The number of chains per unit volume is obtained from eq 2 as

$$
v=\frac{v_{\mathrm{e}}}{h}=\frac{v_{\mathrm{e}}}{\left(1+2 / 3 \dot{\gamma}^{2} \tau_{0}{ }^{2}\right)^{1 / 2}}
$$

The stress component $\sigma_{23}^{*}(\omega, t, \gamma)$ is

$$
\sigma_{23}^{*}(\omega, t, \dot{\gamma})=\frac{v_{\mathrm{e}} k_{\mathrm{B}} T}{h} \frac{i \omega \tau_{0}}{1+i \omega \tau_{0}} a_{0} \mathrm{e}^{i \omega t}
$$

We obtain, from eq 40 , the complex storage and loss moduli corresponding to the periodic part,

$$
\left.\begin{array}{c}
\tilde{G}_{\perp}{ }^{*}(\omega, \gamma)=\frac{v_{\mathrm{e}} k_{\mathrm{B}} T}{h} \frac{i \omega \tau_{0}}{1+i \omega \tau_{0}} \\
\tilde{G}_{\perp}{ }^{\prime}(\omega, \dot{\gamma})=\frac{v_{\mathrm{e}} k_{\mathrm{B}} T}{h} \frac{\omega^{2} \tau_{0}{ }^{2}}{1+\omega^{2} \tau_{0}{ }^{2}} \\
\tilde{G}_{\perp}{ }^{\prime \prime}(\omega, \dot{\gamma})=\frac{v_{\mathrm{e}} k_{\mathrm{B}} T}{h}-\frac{\omega \tau_{0}}{1+\omega^{2} \tau_{0}{ }^{2}}
\end{array}\right\}
$$

The frequency dependence of dynamic moduli is the same as that for linear viscoelasticity; however, the magnitude of the moduli is $\left(1+2 / 3 \gamma^{2} \tau_{0}^{2}\right)^{-1 / 2}$ times as large as that of the linear case.

The zero frequency dynamic viscosity of the periodic part is equal to $\eta(\gamma)$, that is,

$$
\eta_{\perp}(\dot{\gamma})=\lim _{\omega \rightarrow 0} \frac{\tilde{G}_{\perp}{ }^{\prime \prime}(\omega, \dot{\gamma})}{\omega}=\eta(\dot{\gamma})
$$

This is due to the fact that the number of chains has the same value as that in the steady shear flow, though the orthogonal periodic deformation is superposed on the flow. Equation 43, as well as eq 33 , were obtained by using the phenomenological theory ${ }^{19.20}$ and qualitatively verified by experiment. ${ }^{21}$

Acknowledgment. This work was partially supported by a Grant-in-Aid for Scientific Research from the Ministry of Education, Science and Culture of Japan to which we should like to express our cordial appreciation.

\section{REFERENCES}

1. S. Hayashi, Polym. J., 10, 59 (1978).

2. S. Hayashi, J. Soc. Rheol. Jpn., 3, 159 (1975).

3. K. Osaki, M. Fukuda, S. Ohta, and M. Kurata, $J$. Soc. Rheol. Jpn., 2, 106 (1974).

4. N. E. Hudson and Ferguson, Trans. Soc. Rheol., 20, 265 (1976).

5. R. N. Shroff, L. V. Cacio, and M. Shida, Trans. Soc. Rheol., 21, 429 (1977).

6. H. Münstedt, Trans. Soc. Rheol., 23, 421 (1979).

7. H. M. Laun and H. Münstedt, Rhoel. Acta, 15, 517 (1976); ibid., 17, 415 (1978).

8. J. Meissner, Rheol. Acta, 10, 230 (1971).

9. J. Meissner, Trans. Soc. Rheol., 16, 405 (1972).

10. J. Meissner, Rheol. Acta, 14, 201 (1975).

11. T. Raible, A. Demarmels, and J. Meissner, Polym. Bull., 1, 379 (1979).

12. G. V. Vinogradov, A. I. Leonov, and A. M. Proknin, Rheol. Acta, 8, 482 (1969).

13. G. V. Vinogradov, B. V. Radushkevich, and V. D. Fikhman, J. Polym. Sci., A-2, 3, 1 (1970).

14. G. V. Vinogradov, V. D. Fikhman, B. V. Radushkevich, and A. Ya. Malkin, J. Polym. Sci., A2, 8, 657 (1970).

15. H. C. Booij, Rheol. Acta, 5, 215 (1966); ibid., 7, 202 (1968).

16. K. Osaki, M. Tamura, M. Kurata, and M. Kotaka, J. Chem. Phys., 69, 4183 (1965).

17. J. M. Simmsons, Rheol. Acta, 7, 184 (1968).

18. J. W. Sell and W. C. Forman, Macromolecules, 5, 23 (1972).

19. H. Markovitz, "Proceedings of 5th International Congress on Rheology," Vol. 1, S. Onogi, Ed. Maryland University Park Press, Maryland, 1970, p. 499.

20. A. C. Pipkin, Trans. Soc. Rheol., 397 (1968).

21. R. I. Tanner and G. Williams, Rheol. Acta, 5, 215 (1966). 